\title{
Thinking like a lawyer: experiencias didácticas en el aula para fortalecer la formación de un abogado
}

\author{
Thinking like a lawyer: Teaching experiences in the classroom to enhance the formation of \\ a lawyer \\ Dr. Jorge Baraona ${ }^{1}$ \\ Dr. Janet Cádiz ${ }^{2}$ \\ Mg. Olga Villanueva ${ }^{3}$
}

Resumen: La formación del abogado por tradición se ha basado en la manejo de un importante caudal de información de textos doctrinales, con poca o casi ninguna vinculación frente a las situaciones reales que un abogado tendrá que abordar con sus clientes o enfrentar en tribunales.

En directa relación con este tipo de formación se encuentra la manera en que se ha enseñado el Derecho, cuyo acento se ubica en la clase magistral de contenidos más que en reflexiones y prácticas que involucren a dichos contenidos en situaciones reales asociadas a aquello que efectivamente realiza un abogado.

Al considerar lo señalado, el propósito de este artículo es analizar la importancia de una enseñanza del Derecho más proactiva y dinámica y, además, presentar experiencias didácticas en el aula que contribuyan a potenciar la formación de un abogado, desde la perspectiva de una metodología centrada en el aprendizaje. Es así que se describen y analizan evidencias del uso de Aprendizaje Basado en Problemas (ABP), del Método de la Pregunta y del Método del Caso, aplicado a situaciones reales que se presentan en el ejercicio de la profesión de un abogado.

Palabras clave: aprendizaje del Derecho; ABP; método del caso; pensamiento crítico.

Abstract: The formation of a lawyer has been traditionally based on the management of a wealth of information from doctrinal texts, with little or no connection with the real situations that a lawyer will have to deal with in his or her professional life, such as discussing problems face to face with clients or working in the court.

Directly related to this type of training we find that the way in which law has been taught has a marked emphasis on lecture's contents rather than using that content in real situations associated with what actually a lawyer does at work.

Considering the above, the purpose of this article is to analyze the importance of teaching law in a dynamic and proactive way. It also presents classroom learning experiences that contribute to enhance lawyer's formation, from the perspective of a focused learning methodology. Thus, we describe and analyze evidence of the use of Problem Based Learning (PBL), Question Method and Case Method, applied to real situations that arise in practice as a lawyer.

Keywords: Learning law; PBL; Case method; critical thinking.

\footnotetext{
${ }^{1}$ Abogado, Licenciado en Derecho de la PUC, y Doctor en Derecho de la Universidad de Navarra. Ex Decano y actual profesor de la Facultad de Derecho de la Universidad de los Andes (Chile) 2010-2012. jbaraona@jbcia.cl.

${ }^{2}$ Licenciada en Educación, Universidad Católica Raúl Silva Henríquez//IPES Blas Cañas/ Magíster en Ciencias de la Educación, Mención Evaluación, Pontificia Universidad Católica de Chile, Ph D in Education Research, Methodology Evaluation and Measurement, University of California, Los Angeles, UCLA. jcadiz@uandes.cl

${ }^{3}$ Magister en Ciencias de la Educación de la Pontificia Universidad Católica de Chile, Licenciada en Psicología y Pedagogía de la Universidad Pedagógica de Bogotá, profesor Universidad de los Andes. ovillanueva@uandes.cl
} 


\section{Formación de un abogado en los tiempos actuales: un análisis prospectivo.}

Es altamente probable que al considerar una aproximación a lo que significa la formación del abogado, sea inevitable remitirse a una percepción preconcebida respecto de un sujeto cuya permanencia en el espacio académico está directamente relacionada con su capacidad de escucha y posibilidad de memorización de gran cantidad de información, la cual además, de ser posible, debe llegar a recitar como si se tratase de la más básica de las tablas de multiplicar. Así "la clase se llena, generalmente, con una recitación del profesor, solo incidentalmente interrumpida por alguna pregunta o solicitud de aclaración de algún alumno"4 poca participación por parte del estudiante en la dinámica que se desarrolla durante la clase.

Lo anterior, no es más que la evidencia de un reiterativo proceso educativo en el cual por tradición el énfasis se encuentra centrado en aquello que es trasmitido en forma expositiva por el docente y no en la capacidad de comprensión, análisis, síntesis y reflexión lógico-jurídica por parte del estudiante. Dicho proceso educativo es comprendido como aquel espacio en el cual, lo trascendental es la enseñanza y no el aprendizaje, es decir, aquello que tiene primacía es lo que se encuentra relacionado mucho más con la labor del docente que con la acción participativa del estudiante. Al respecto: "La enseñanza tradicional del derecho se ubica casi exclusivamente en el área de la exposición oral, con el consabido detrimento y limitación de otros métodos y estrategias (...) busca preferentemente transmitir un cúmulo de información referente al contenido de las normas jurídicas, así como de los elementos necesarios para que el alumno pueda interpretar esas normas"5.

Lo anterior, deja de manifiesto un evidente vacío en la preparación de las nuevas generaciones de abogados en tanto no se incluya una diversificación de las formas en las cuales puede ser enseñado el Derecho en todas sus vertientes.

De acuerdo con lo anterior, la forma de enseñar el Derecho, ha estado marcada por una hegemónica preparación basada mucho más en aquello que debe ser trasmitido al alumno que en lo que se convertirá en un aprendizaje relevante para él. Por tanto, debe ser una labor primordial del docente asumir una postura de mediador entre el aprendizaje y el alumno, de manera tal que este último, pueda acercase como futuro profesional de las leyes a una nueva manera de abordar su profesión.

En consecuencia se espera que el docente, al desarrollar el proceso de enseñanza aprendizaje, sea capaz de desarrollar un trabajo con sus estudiantes en donde logre: "Impulsar y promover todas las exploraciones, experiencias y proyectos que éstos preferentemente inicien o decidan emprender (...) entendiendo que los alumnos son entes individuales, únicos y diferentes de los

\footnotetext{
${ }^{4}$ GONZÁLEZ, Raimundo. "Devenir y porvenir del Derecho Espacial”. Elementos de juicio revista de temas constitucionales. 2007, núm. 5. Año II. P. 61-78.

${ }^{5}$ ESPINOZA, Francisco. "Métodos y estrategias para la enseñanza-aprendizaje del derecho". International Journal of Good Conscience. 2009, vol. 4, núm. 1. P. 31-74.
} 
demás, seres con iniciativa, con necesidades personales de crecer, capaces de autodeterminación y con la potencialidad de desarrollar actividades y solucionar problemas creativamente ${ }^{6}$.

Además, como complemento de lo anterior, el profesor "debe ser un facilitador de la capacidad potencial de autorrealización de los alumnos, sus esfuerzos didácticos deben encaminarse a lograr que las actividades de los alumnos sean autodirigidas y fomenten al autoaprendizaje y la creatividad"7. Todo esto, en el marco de una visión formativa que considera la movilidad y los cambios que se producen naturalmente entre las distintas generaciones de abogados.

Por otro lado, es ampliamente sabido que la manera en la que el docente acerque al estudiante al conocimiento durante el desarrollo de su clase, dependerá en gran medida no solo de su forma particular de entender lo que le es propio a la preparación del abogado, sino que también está demarcado por sus propias experiencias de formación, su capacidad de innovación y su posibilidad de acercamiento a una visión pedagógica de la enseñanza-aprendizaje de la abogacía.

Es por lo antes planteado, que se pueden observar comentarios emitidos por docentes-abogados reconociendo lo siguiente: "Olvidamos enseñar los procesos, las prácticas discursivas que nosotros mismos hemos ido adquiriendo a lo largo del tiempo, la urdimbre con la que construimos la ciencia. Organizar las clases sólo desde una estrategia de aprendizaje, esencialmente las clases magistrales, conduce a olvidar que el conocimiento de los estudiantes ha de adquirirse del mismo modo que nos han formado a nosotros, aprendiendo los modos de indagar e investigar, de hacernos las preguntas adecuadas, de sortear entre múltiples opiniones hasta alcanzar una propia ${ }^{8}$.

Al considerar lo antes planteado, es lógico pensar que en el contexto educativo del abogado se prioriza el uso casi tradicional e irrefutable de una única manera de llevar a cabo el proceso de enseñanza aprendizaje, la cual es a través de la ya reconocida clase magistral, esta estrategia empleada por la gran mayoría de los docentes-abogados, es quizás el método educativo más usado aún en la actualidad y refleja el modelo didáctico preferido por los docentes de distintas áreas.

Respecto de lo anterior, es importante indicar que si bien la clase magistral es el método más empleado en la enseñanza del derecho, desde el punto de vista pedagógico no es necesariamente es el más efectivo para lograr aprendizajes profundos en los estudiantes. En esta línea: "La cátedra magistral tiene una arraigada tradición en las escuelas y facultades de derecho. Este modelo de enseñanza privilegia el discurso de los profesores, y en la mayoría de las ocasiones provoca pasividad en los estudiantes, pues resulta poco propicio para incentivar su participación en clase; además, induce a la memorización de datos por encima de una labor analítica de la

\footnotetext{
${ }^{6}$ ESPINOZA, Francisco. "Métodos y estrategias para la enseñanza-aprendizaje del derecho". International Journal of Good Conscience. 2009, vol. 4, núm. 1. P. 53.

${ }^{7}$ Ibid.

${ }^{8}$ SÁNCHEZ-ARCILLA, José y MADRID, María. "Una propuesta de nuevas estrategias para la enseñanza del derecho”. Revista de educación y derecho. 2010, núm. 1. P. 1-25.
} 
información e inhibe la actitud crítica de los estudiantes frente a las afirmaciones que formulan los docentes ${ }^{9}$.

De acuerdo con lo anterior, no cabe la menor duda respecto de que el uso de la clase magistral como estrategia para la formación de los abogados, es poco favorable y puede generar un escaso aporte para el desarrollo de habilidades superiores en el alumno. Sin embargo, también es importante reconocer que como parte de la metodología de trabajo del docente, es recomendable el uso de la clase magistral, siempre y cuando se emplee como base para la trasmisión de contenidos puntuales y relevantes a partir de los cuales puedan sustentarse las acciones prácticas asociadas a dichos contenidos.

Como consecuencia inmediata, es importante aclarar que, actualmente, en la formación de abogados se evidencia una marcada preocupación por la renovación del sistema que se encarga de educar a los futuros profesionales del Derecho, en donde se hace cada vez más evidente la necesidad de proveer experiencias educativas que le permitan al estudiante dejar de ser un sujeto pasivo y convertirse en un ser partícipe de su aprendizaje a través del desarrollo de pensamiento crítico, reflexivo y resolutorio de problemas, que le estimule y habilite no sólo a comprender aquello que teóricamente le es trasmitido, sino que además le confiera la posibilidad de extrapolarlo a situaciones reales a las que muy probablemente se verá enfrentado a lo largo de su vida profesional.

Además de lo antes señalado, quizás el principal punto de quiebre a partir del cual puede justificarse la preocupación por optimizar la forma particular como han sido impartidas hasta hoy las cátedras de Derecho, es la evidente distancia que existe entre la teoría y la práctica, lo cual se refleja en las distintas situaciones a las que se ven enfrentados los abogados recién graduados, a quienes al ingresar al contexto laboral, les exigen un sin número de competencias, habilidades y conocimientos para los que en ocasiones no se encuentra realmente preparados.

En forma puntual, "la enseñanza jurídica actual es mínimamente práctica, se prefiere aprender la norma en abstracto, perdiéndose la oportunidad de obtener y desarrollar capacidades, aptitudes, habilidades y destrezas que atiendan los hechos y problemas prácticos que resuelven los abogados postulantes, los procuradores de justicia y los administradores de justicia"10. Lo cual no es posible de aprender sólo a través del manejo de los contenidos. Es por ello que "el complemento de las clases teóricas son las clases prácticas, dedicadas generalmente a la propuesta, resolución y discusión de casos prácticos extraídos de la imaginación del profesor, de la actualidad o, más frecuentemente, de la jurisprudencia" ${ }^{\text {"1 }}$ en donde el estudiante debe emplear nuevas formas de aproximarse al conocimiento.

Ahora bien, es relevante considerar que el acortamiento de la brecha entre aquello que se enseña en forma teórica y su posibilidad de aplicación al ámbito práctico, requiere entre otras cosas, de una nueva manera de entender la labor del abogado y de un cambio en la mentalidad tanto de

\footnotetext{
${ }^{9}$ CÁRDENAS, María. Ensayo sobre didáctica y pedagogía jurídica. [Consultado en mayo de 2015]. Disponible de web http://biblio.juridicas.unam.mx/libros/5/2406/9.pdf

${ }^{10}$ ESPINOZA, Francisco. "Métodos y estrategias para la enseñanza-aprendizaje del derecho". International Journal of Good Conscience. 2009, vol. 4, núm.. P. 33.

${ }^{11}$ Ibid. P. 59.
} 
académicos como de alumnos, pues son ellos quienes deben generar la modificación conductual, la que parte por una nueva percepción acerca del aprendizaje del Derecho.

Considerando lo anterior, es posible comprender entonces que "se requiere un cambio de comportamientos y actitudes no solo de docentes, sino de dicentes, que entre otras cosas superen viejas ideas de que se aprueba la asignatura con tan solo memorizar unos apuntes días antes del eventual control final"12. Dicho cambio reflejaría una aproximación inicial a una nueva visión de formación de abogados en la cual se genere apertura a estrategias didácticas en las que se potencien habilidades reconocidas por el docente como necesarias para el ejercicio práctico de la profesión, reconociendo la importancia de "los métodos y estrategias didácticas como procedimientos utilizados por el profesor con la finalidad de producir en los alumnos determinadas experiencias de aprendizaje" ${ }^{\prime 13}$, las cuales el alumno percibe con un sentido de aplicabilidad.

Todo lo anterior, permite reconocer que la enseñanza del derecho puede ser optimizada en la medida que se considere que: "Ejercitar al estudiante en el conjunto de técnicas, de métodos y de procedimientos que le permitan conocer el Derecho por sí mismo, identificar y resolver por sí mismo los problemas que plantea su interpretación y aplicación y formular por sí mismo las soluciones que pueden solventarlos (...) Le permitirá establecer una conducta que refleje su pensamiento como abogado, pues el docente "más que facilitar directamente el contenido teórico que debe retenerse, deberá hacer transparente y reproducir ante el alumno el procedimiento, el camino a través del cual él mismo podría llegar a su formulación. Ello implica familiarizarlo con los procedimientos de análisis, síntesis, relación de conceptos, desarrollo de contenidos, formulación de conclusiones, expresión verbal de posiciones, desarrollo de debates, etcétera." 14

Por tanto, la ganancia de un sistema de formación centrado en el aprendizaje del alumno y el desarrollo de sus habilidades, permite configurar en él pensamiento contextualizado como abogado, que le permitirá responder positivamente a lo que se espera de su desempeño como un operador de la profesión legal.

Todo lo antes mencionado, permite introducir de manera general la propuesta de formación de abogados llevada a cabo, de manera experimental, en la Facultad de Derecho de la Universidad de los Andes, la cual partió su ciclo de implementación durante el año 2011 y que buscó incorporar al proceso de enseñanza aprendizaje, tres distintas estrategias didácticas: el Aprendizaje Basado en Problemas (ABP); el Método de la Pregunta; y el Método del Caso. En la siguiente sección, se describen estas estrategias didácticas.

\footnotetext{
12 PEÑA, Salomé. “Técnicas didácticas en la enseñanza del Derecho del Trabajo" [en línea]. Jornada sobre la Enseñanza del Derecho del Trabajo en el Espacio Europeo de Enseñanza Superior. 2011 [Consultado en mayo 2015]. Disponible en web http://fundacion.usal.es/aedtss/images/stories/Pea.pdf

${ }^{13}$ ESPINOZA, Francisco. "Métodos y estrategias para la enseñanza-aprendizaje del derecho". International Journal of Good Conscience. 2009, vol. 4, núm. 1. P. 33.

${ }^{14}$ Ibid. P. 1.
} 


\section{Experiencias didácticas que contribuyen a fortalecer la formación de un abogado}

Diversas son las estrategias didácticas que hoy en día se emplean en la formación de distintos tipos de profesionales y la enseñanza del Derecho no difiere de ello, por cuanto se encamina a una visión en la cual se espera que el estudiante pueda acercase al mundo del trabajo de modo más directo y real. Es así que estrategias didácticas tales como el Aprendizaje Basado en Problemas (ABP), el Método de la Pregunta y el Método del Caso, entre otras más, hoy se están incorporando a las distintas asignaturas.

\section{1.- Aprendizaje Basado en Problemas (ABP).}

\section{a) Características principales}

Desarrollado y llevado a la práctica en los años 60's en las escuelas de medicina de las Universidades de Mc Master en Canadá y de Case Western Reserve en los Estados Unidos, esta es una estrategia educativa que permite desarrollar en el alumno razonamiento y juicio críticos, a partir de la estimulación del aprendizaje desde el conflicto cognitivo que debe enfrentar el estudiante al analizar cada una de las situaciones problemáticas que se le presentan.

En consecuencia, entre las principales capacidades que se potencian con el ABP están: adquisición del razonamiento crítico; habilidad para escuchar, responder y participar en discusiones relevantes; oportunidad de aprender a tomar decisiones, aprendizaje auto-dirigido y capacidad para el trabajo en equipo.

Esta estrategia didáctica puede ser aplicada desde los primeros años de formación de un abogado, sin embargo, en los semestres más avanzados de la formación, se recomienda que se aplique no sólo en forma grupal sino también individual como una manera de fortalecer la autonomía. Por su parte, al trabajar de manera grupal, se incentiva el trabajo colaborativo y el liderazgo, lo cual resulta relevante si se piensa que en la práctica muchas veces un abogado coexiste junto a otros abogados para resolver distintas situaciones.

\section{b) Modelo metodológico del "ABP" aplicado en cursos de la Facultad de Derecho de la Universidad de los Andes}

El modelo metodológico diseñado para implementar la estrategia didáctica ABP, admite 6 pasos fundamentales: la presentación de la situación problemática en el contexto del Derecho; los hechos, pistas y datos; el problema; las fuentes de información; las estrategias de solución y manejo de conflictos; y las conclusiones.

En términos sintéticos, el paso $\mathrm{n}^{\circ} 1$ implica que los alumnos lean la situación problemática para familiarizarse con el contexto y actores que participan en el "Ejercicio ABP", de forma individual, para luego hacerlo en forma grupal, donde se intercambian las primeras impresiones.

El paso $\mathrm{n}^{\circ} 2$ busca que el equipo de estudiantes determine y discrimine lo esencial de lo accesorio al caso. Para ello, deben identificar y diferenciar los hechos primarios de los secundarios, así 
como los actores claves y los que tienen una participación relativa en el problema que se está presentando.

En cuanto a los pasos 3, 4 y 5 son aquellos en los que se revela y analiza la problemática, fundamentándola a través de la norma y la jurisprudencia, hasta llegar a soluciones plausibles.

Por último, el paso $\mathrm{n}^{\circ} 6$ contribuye a concluir el análisis realizado, incluyendo una reflexión acerca de la utilidad de la estrategia para el desarrollo profesional de un abogado.

\section{c) Sobre las experiencias reportadas del uso del "ABP"}

El efecto que tuvo la aplicación de la estrategia ABP en los estudiantes de Derecho, logra visualizarse en los comentarios emitidos por estos, en donde se valora la instancia de reflexión ante problemas reales que van a tener que enfrentar en más de una ocasión en su quehacer profesional. También se hace notar las diferencias que se producen ante una enseñanza tradicional del Derecho versus una que los ayude a asumir su rol de abogados desde su proceso de formación y no tiempo después del egreso.

Los testimonios recolectados evidencian con claridad la importancia del ABP como una instancia pedagógica que valoran los alumnos, en donde a través de sus análisis, el profesor también se puede dar cuenta que sus estudiantes no son un ente pasivo, sino activo que requiere estímulos e incentivos pedagógicos tales como los que les proveen estrategias como el ABP.

\section{2.- Método de la Pregunta.}

\section{a) Características principales del Método de la Pregunta}

El método de la pregunta forma parte de las estrategias empleadas para el fomento del pensamiento crítico. Su principal objetivo es, literalmente, hacer pensar al estudiante a través de interrogantes que no dependan de un "sí" o un "no", sino que vayan dándole al estudiante la oportunidad de ser cuestionado y de que el mismo se cuestione respecto de aquello que se le presenta como verdad, situación o problema.

En el caso particular de la experiencia realizada en cursos de Derecho Civil y Derecho Constitucional, el método de la pregunta se empleó para orientar la profundización en los contenidos abordados en las clases, a través de la resolución de interrogantes asociadas a los conocimientos claves de cada una de las unidades de aprendizaje planteadas en los programas de curso.

De esta forma, los estudiantes tuvieron la oportunidad de potenciar su aprendizaje, al darse cuenta que cada unidad de aprendizaje, correspondería al develamiento de interrogantes sustantivas para una mejor comprensión y aplicación de dichos contenidos. Se destaca, además, que estas interrogantes se enmarcaron en un contexto real y cercano a la profesión del abogado, por lo tanto, no se centraron en la materia por la materia, sino que en el uso de ese contenido aplicado a situaciones más cercanas al contexto profesional. 


\section{b) Modelo metodológico del 'Método de la Pregunta' aplicado en cursos de la Facultad de Derecho de la Universidad de los Andes}

En el caso del "Método de la Pregunta", el modelo metodológico diseñado para implementar la estrategia, consideró la revisión preliminar de 3 elementos relevantes: el programa de curso; el syllabus del curso y el plan de trabajo. Con toda la información recabada desde estas fuentes, se dio paso a la construcción de una guía de preguntas.

El propósito al emplear esta estrategia estaba centrado en la búsqueda de "coherencia pedagógica", entre lo que se propone en el programa, lo que se planifica en el syllabus y plan de trabajo y lo que se termina trabajando con los alumnos en la sala de clases. Principalmente se apuesta a un desarrollo del pensamiento crítico, en donde las preguntas actúan como un medio para situarse en el ejercicio de la profesión.

\section{c) Sobre las experiencias reportadas del uso 'Método de la Pregunta'}

Básicamente, la primera apreciación de los alumnos es que - en apariencia - esto puede ser más trabajo, al encontrarse con una guía de preguntas que los obliga, de cierto modo, a preparar la materia antes, durante y después de la clase. Sin embargo, una vez que se observa que las preguntas orientan la clase y facilitan la discusión comienza a tener más sentido para los alumnos, los cuales muchas veces tampoco estaban acostumbrados a ser co-participes de las clases.

\section{3.- Método del Caso.}

\section{a) Características principales del 'Método del Caso'}

Su incursión en el ámbito del derecho se remonta al año 1870 y es el Prof. Christopher Langdell, ex Decano la Harvard Law School, donde lo introduce, buscando una renovación de la enseñanza del Derecho en los Estados Unidos, y paradójicamente muy influido por una visita que hizo a las universidades alemanas. Es una estrategia didáctica cuya finalidad es enfrentar al alumno de derecho a situaciones reales, frente a las cuales, debe tomar decisiones, valorar y consensuar acciones y emitir juicios críticos fundamentados.

Es una de las estrategias didácticas más completas y de real cercanía a las prácticas de un abogado, siendo por ello necesario que el profesor, que va a emplearla, seleccione cuidadosamente los casos, de manera que en cada una de ellos se propongan desafíos cognitivos de alto nivel para los alumnos.

\section{b) Modelo metodológico del 'Método del Caso' aplicado en cursos de la Facultad de Derecho de la Universidad de los Andes}

En cuanto al modelo metodológico que se diseñó e implementó, éste se define con tres pasos fundamentales, a saber: antecedentes y/o hechos del caso o la sentencia; aplicación de normas del derecho y principios de justicia; y fallo/resolución del caso. 
Todo lo anterior, se complementa a su vez con preguntas claves para cada uno de los pasos antes mencionados. Por ejemplo, en el paso 1 se plantearon interrogantes tales como: En función de la estructura de la sentencia, ¿qué secciones de la sentencia corresponden a la parte expositiva, considerativa y resolutiva, respectivamente?; ¿cuáles son las partes que se encuentran involucradas en el caso que se presenta la sentencia? y ¿qué tipo de relación existe entre las partes?; entre otras. Respecto al Paso 2 se destacan, a nivel de ejemplo: ¿qué norma o normas jurídicas se aplicaron para la determinación de los hechos o aspectos controversiales expuestos en el caso de la sentencia?; ¿qué juicio crítico cabe realizar a la estrategia procesal que han utilizado las partes?; ¿lo habría(n) efectuado de otra forma?; y si es así, ¿de qué otra manera? Por último, en el Paso 3 se consideraron interrogantes como: ¿qué juicio crítico tiene (n) respecto al criterio probatorio aplicado por el tribunal o juez, desde la perspectiva "legal" y de la "sana crítica"?; o bien, Conforme con el "Fallo" dictado por el tribunal o juez, ¿está(n) de acuerdo con este "Fallo", en función de la controversia o pleito presentado y las partes involucradas?. Estas interrogantes más otras incluidas en el desarrollo de la estrategia, contribuyen a configurar un cierre del caso en cuanto a la sentencia estudiada y sus implicancias.

Lo clave de estos pasos que se acompañan de interrogantes es que ayudan a guiar el pensamiento analítico y crítico del futuro abogado, permitiendo de este modo que centre sus focos de interés en el caso desde una perspectiva integral, basada en el saber, en el saber hacer, en el saber ser y en el saber convivir. Esto último se refuerza en la estrategia, a partir del trabajo colaborativo que deben realizar los alumnos que conforman el equipo de abogados que se hacen cargo del caso, en términos de simulación pero con una vivencia real.

\section{c) Sobre las experiencias reportadas del uso del 'Método del Caso'}

Sin duda alguna, el 'Método del Caso' es una de las estrategias didácticas más completas desde el punto de vista de las destrezas y habilidades que logra fomentar y desarrollar en los alumnos que se están formando para ejercer como abogados. Lo anterior, se evidencia en los comentarios emitidos por profesores y estudiantes quienes luego de haber participado en este tipo de experiencias, reconocieron las ventajas de emplear esta estrategia. En forma específica plantearon lo siguiente:

- Permite a los alumnos analizar y cuestionarse sobre casos reales de la jurisprudencia.

- Contribuye a que los alumnos puedan evaluar los principios o reglas de justicia frente al caso que se esté analizando, a través de un razonamiento crítico.

- Fomenta la capacidad para resolver problemas, a partir de un caso jurisprudencial.

- Fortalece las destrezas y habilidades necesarias para analizar los criterios probatorios de una sentencia.

- Posibilita la realización de un trabajo autónomo y colaborativo, tal como se hace en una oficina de abogados.

En definitiva, contribuye a desarrollar un conjunto de competencias que permitan evaluar las sentencias y el tenor específico de los casos, de forma integral, tanto analítica como 
sintéticamente, de cara a obtener una postura fundamentada frente a la resolución de un caso y los efectos ético-morales que en este marco pudieran presentarse.

\section{Conclusiones}

De acuerdo a lo abordado en este artículo, hoy en día se requiere de un profundo compromiso por lograr que el futuro abogado se forme en las aulas a través de un aprendizaje activo-prácticocrítico. Es decir, donde lo meramente cognitivo, se ensamble con la necesidad de desarrollar en los alumnos habilidades que son indispensables para que como abogados puedan desempeñarse eficazmente en contextos laborales diversos.

Es así que, por ejemplo, la expresión oral y escrita adecuadas, capacidad de análisis y de síntesis, razonamiento jurídico bien estructurado, capacidad de redacción de diversos instrumentos legales, habilidad para investigar certeramente en doctrina y jurisprudencia, capacidad de trabajo colaborativo, liderazgo, habilidad de negociación, etc.. Todas ellas son competencias que no pueden quedar entregadas al devenir de una clase unidireccional, donde interesa únicamente cubrir los contenidos, y hay un cierto desinterés por apropiarse críticamente de ellos, para así lograr las destrezas y habilidades que impliquen formar a un abogado competente en su hacer, en su ser y en su saber.

Lo anterior supone una clase más "compleja", donde el profesor ya no está preocupado de sólo abordar contenido, sino de preparar una clase que provoque la atención del alumno, motivándolo a que sea co-partícipe de su proceso integral de formación. Lo anterior, permite evidenciar que la labor del docente empieza a adoptar un matiz diferente, dado que requiere la inclusión de cambios metodológicos, en particular, en la manera como son planificadas y desarrolladas las clases; así como también el modo en que son evaluadas.

En función de esto, se abre para el docente un espectro de posibilidades de actuación sobre la base de diversas estrategias didácticas que le permitan conformar su propio modelo de formación, tales como las que se mencionaron en la sección precedente. Sobre este punto es importante que "cada modelo didáctico, legitima un discurso diferente del profesor y hay varios estilos. Por ello, es preciso reconocer la relación entre el propio discurso didáctico, en tanto van interactuando con el discurso jurídico y la puesta en práctica de todo ello, ya que no será igual el que expone principios y normas generales de códigos, leyes, doctrina, jurisprudencia y el que se enseña a través del estudio de casos" $"$.

En último término, una nueva manera de formar al futuro abogado implicará, sin duda, una nueva manera de enseñar, por tanto, será un compromiso a adquirir por parte de las instituciones que hoy se dedican a preparar a los abogados del siglo XXI.

15 De BIANCHETTI, Alba. "La enseñanza del Derecho". [Consultado en mayo 2015]. Disponible en web http://www.unne.edu.ar/unnevieja/Web/cyt/cyt/2002/01-Sociales/S-053.pdf 


\section{Referencias bibliográficas}

CÁRDENAS, María. Ensayo sobre didáctica y pedagogía jurídica. [Consultado en mayo de 2015]. Disponible de web http://biblio.juridicas.unam.mx/libros/5/2406/9.pdf

De BIANCHETTI, Alba. "La enseñanza del Derecho". [Consultado en mayo 2015]. Disponible en web http://www.unne.edu.ar/unnevieja/Web/cyt/cyt/2002/01-Sociales/S-053.pdf

ESPINOZA, Francisco. "Métodos y estrategias para la enseñanza-aprendizaje del derecho". International Journal of Good Conscience. 2009, vol. 4, núm. 1. P. 31-74.

GONZÁLEZ, Raimundo. "Devenir y porvenir del Derecho Espacial”. Elementos de juicio revista de temas constitucionales. 2007, núm. 5. Año II. P. 61-78.

SÁNCHEZ-ARCILLA, José y MADRID, María. "Una propuesta de nuevas estrategias para la enseñanza del derecho". Revista de educación y derecho. 2010, núm. 1. P. 1-25.

PEÑA, Salomé. “Técnicas didácticas en la enseñanza del Derecho del Trabajo" [en línea]. Jornada sobre la Enseñanza del Derecho del Trabajo en el Espacio Europeo de Enseñanza Superior. 2011 [Consultado en mayo 2015]. Disponible en web http://fundacion.usal.es/aedtss/images/stories/Pea.pdf 